\title{
Ballpark Figure Algorithms for Data Broadcast in Wireless Networks
}

\author{
K.S.Narmatha \\ Department of Computer Science and Engineering, \\ Bharathidasan University, Tiruchirappalli, \\ Tamil Nadu, India
}

\author{
E.GeorgeDharmaPrakash Raj \\ Department of Computer Science and Engineering, \\ Bharathidasan University, Tiruchirappalli, \\ Tamil Nadu, India
}

\begin{abstract}
In wireless system allocation is a necessary purpose and show industry expensive dependability in message protocol design. In multihop wireless networks, equally, imposition by a node by reason of immediate transmissions as its neighbors makes it nontrivial to graph a minimum-latency transmit algorithm, which is known to be NP-complete. A simple ballpark figure algorithm for the one-toall transmit problem that improves all previously documented guarantees for this problem. In All-to-all transmit problem where every node sends its own consequence to all complementary nodes. In the all-to-all transmit problem, we current two algorithms with ballpark figure ratios of 20 and 34, civilizing the greatest result. A communication wants to be transmitted establishment its resource to all the previous nodes in the network. There may be different messages to be broadcasted from several sources. Two or more nodes broadcast a communication to an ordinary neighbor at the same time; the frequent node will not collect any of this communication. We say that collide has occurred at the ordinary node. So any message protocol for wireless networks must dispute with the distress of difficulty in the wireless intermediate.
\end{abstract}

Keywords: Adhoc network; Ballpark algorithm; Transmit algorithm; wireless scheduling;

\section{INTRODUCTION}

Wide broadcasting is a necessary development in wireless networks, in which a message requirements to be transmitted from its resource to all the further nodes in the network. There may be different communication to be broadcasted from multiple sources. some network protocols rely on broadcasting, for example, in sequence dissemination, package/source improvement, or direction-finding in multihop wireless networks demanding that type applications of multihop wireless networks consist of not a success discharge and release operations forces message, and exclusive of interruption entity detection using sensors, the propose of low-latency broadcasting process is required to meet thorough end-to-end intermission supplies for higher-level applications. Imposition is an innovative qualified problem in wireless networks. When two or more nodes broadcast a communication to a wide-ranging neighbour at the equivalent time, the regular node will not recognize any of this communication. In such a case, we say that crash has occurred at the regular node. Invasion group may be continuous better than the broadcast range, in which case a node may not collect a communication from its source if it's within the interruption range of a different node sending a message. The design of low-latency broadcasting system is necessary to meet tough end-to-end impediment requests for higher-level applications. When two or more nodes broadcast a communication to a regular neighbor at the equivalent time, the regular node will not collect some of this communication. Interfering range may be constant larger than the broadcast range, in which case a node may not collect a communication from its receiver.

\section{RELATED WORK}

The algorithms for ONE-TO-ALL and ALL-TO-ALL broadcasting problems. In one-to-all broadcast, there is a source that sends a message to all other nodes in the network. In all-to-all transmit each node sends its individual communication to all supplementary nodes .constant the oneto-all broadcasting problem is known to be NP-complete. For in cooperation problems, we increase ballpark figure algorithms, which develop the preceding consequences. For ONE-TO-ALL transmit problem, we convenient a simple ballpark figure algorithm that achieves a ballpark solution, thereby recovering the ballpark figure assurance. Our algorithm is based on following two ideas that lead to the development: 1) dispensation the nodes acquisitively in no growing order of the quantity of receivers, and 2) allowing nodes to broadcast more than once. The latter is principally counter-intuitive as one would be expecting that the latency would enhance if a node transmits less than once. Note that in the difficult of their algorithm gives an ballpark figure assurance that is greater than 400 .

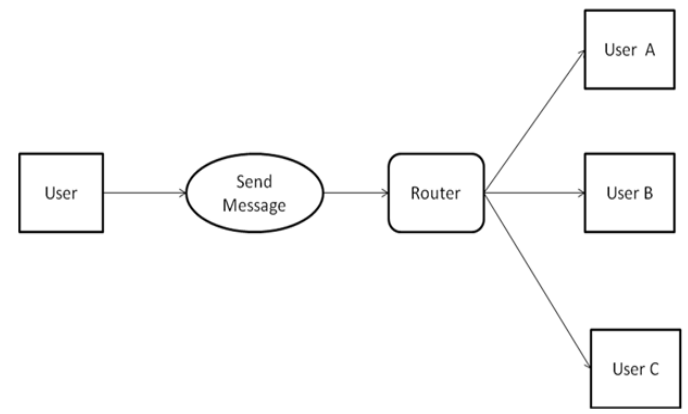

Fig 1.One to All Broadcast

ALL-TO-ALL BROADCAST problem and current two algorithms (called CDA and ICDA) with ballpark figure guarantees of 20 and 34, correspondingly, thereby recovering the ballpark figure guarantee of 27. Our enhanced product is due to well-organized development technique to collect data and then perform pipelined broadcasting. In ICDA, all nodes are scheduled to contribute in transmissions as before time as 
feasible. Constant although its hypothetical bound is weaker than that of CDA, untried results prove that it afford improved presentation.

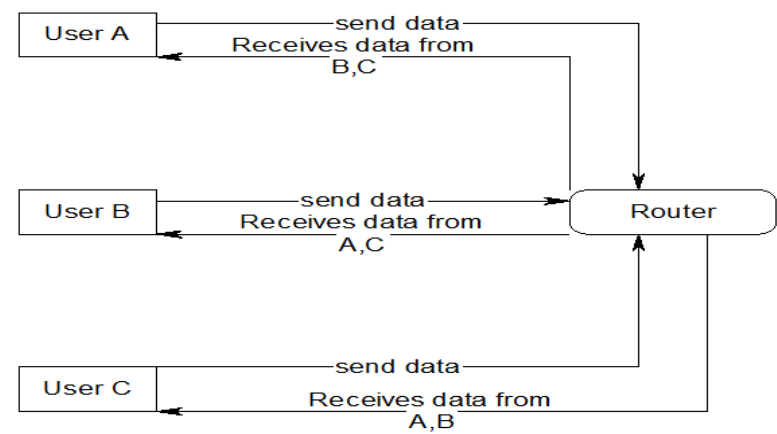

Fig 2.All to All Broadcast

We then reflect on the all-to-all transmit problem where every node sends its own communication to each and every one further nodes. Civilizing the most excellent consequence accessible in the text, we current two algorithms with ballpark figure ratios of 20 and 34. Algorithms construct greatly enhanced in observe than the worst-case guarantees provided in the hypothetical investigation and complete it gives 37 percent presentation development greater than obtainable schemes.

\section{SYSTEM MODULE}

\subsection{Ad Hoc-Networking}

Ad-hoc network is a local area network (LAN) that is built spontaneously as devices connect. Instead of relying on a base station to coordinate the flow of messages to each node in the network, the individual network nodes forward packets to and from each other. In Latin, ad hoc literally means "for this," meaning "for this special purpose" and also, by extension, improvised. In the Windows operating system, ad-hoc is a communication mode (setting) that allows computers to directly communicate with each other without a router.

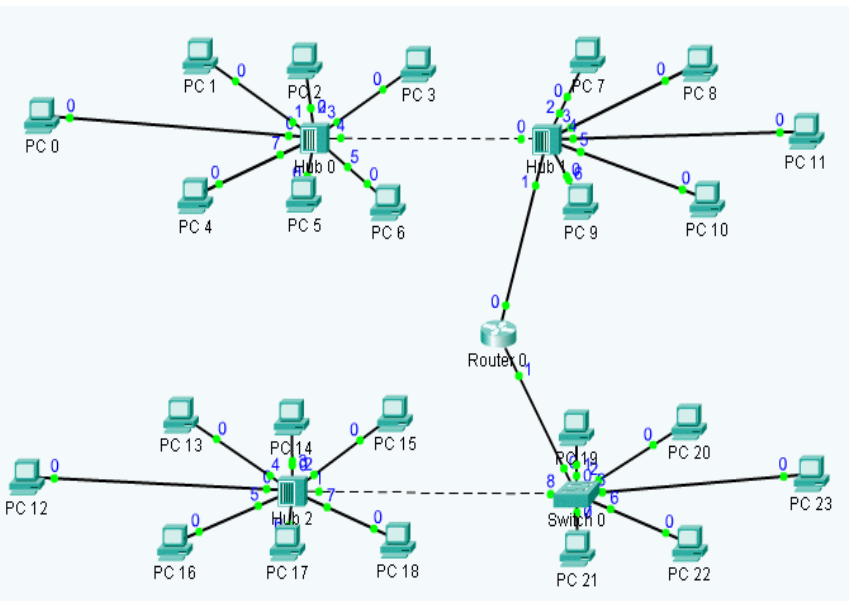

Fig 3.System Module
Ballpark figure algorithms are algorithms used to find ballpark solutions to optimization problems. Ballpark figure algorithms are often connected with NP-hard inconvenience; because it is improbable that around be capable to increasingly be well-organized polynomial time exact algorithms solving NP-hard problems, one settles for polynomial decentralized type of wireless networkable time sub-optimal solutions. Unlike heuristics, which usually only find realistically high-quality solutions realistically quick, one requirements demonstrable solution value and demonstrable run time limits. if possible, the ballpark figure is best possible up to a small constant reason (for instance within $5 \%$ of the optimal solution). ballpark figure algorithms are progressively more being used for effort where demand polynomial-time algorithms are recognized but are too high-priced due to the contribution range. A usual example for a ballpark figure algorithm is the one for peak envelop in graphs: find an discovered frame and include equally endpoints to the peak envelop, pending nobody wait. It is comprehensible that the consequential envelop is on generally two times because outsized as the best possible one. This is a even reason ballpark figure algorithm with a reason of 2

\subsection{Transmit Algorithm}

Transmit algorithms are typically executed concurrently, with separate parts of the algorithm being run simultaneously on independent processors, and having limited information about what the other parts of the algorithm are doing. One of the main challenges in initial and implementing disseminated algorithms is profitably coordinating the performance of the autonomous parts of the algorithm in the features of workstation failures as well as changeable exchanges links. The preference of a correct disseminated algorithm to work out a specified difficulty depends scheduled equally the quality of the difficulty, and quality of the method the algorithm will run scheduled such as the category with possibility of workstation or connection failures, the kind of inter-process message that can be performed, and the intensity of time organization involving disconnect processes.

\subsection{Wireless Scheduling}

How capacity is shared between users in a network .A set of rules that specify which user is allowed to transmit and which user is allowed to receive at each time-slot. In real-life systems we have to assume a delay because the scheduler.

\section{Collects path estimates}

Takes a development result

Notify the particular customer

Assumes that a wonderful path estimate is accessible at time $t+\tau$

\subsection{Ballpark Figure Algorithm}




\section{EXPRIMENT RESULT}

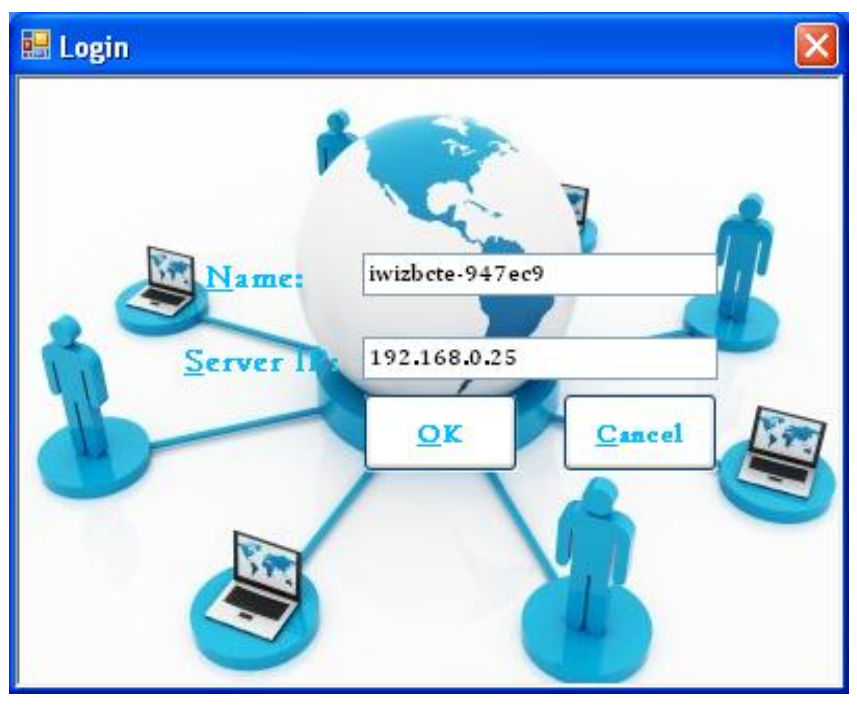

Fig 4.login window

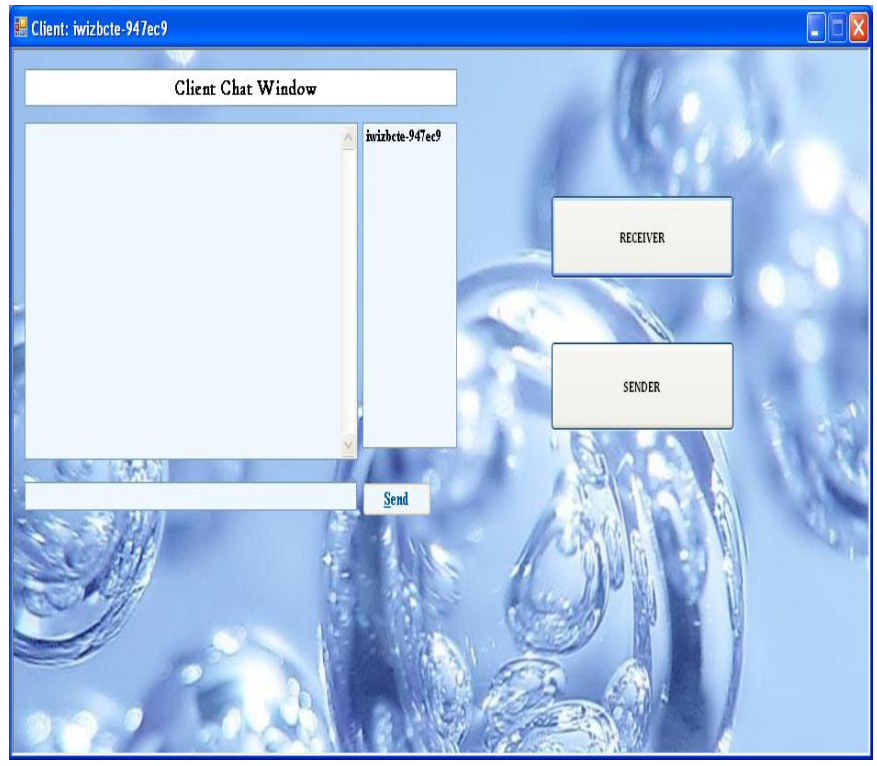

Fig5.ClientChatwindow

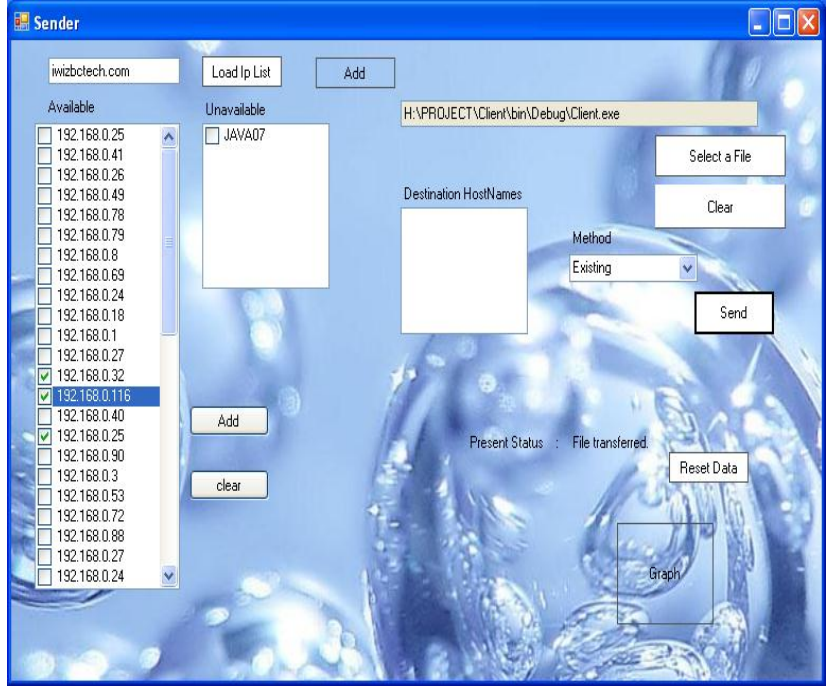

Fig 6.Sender select the path

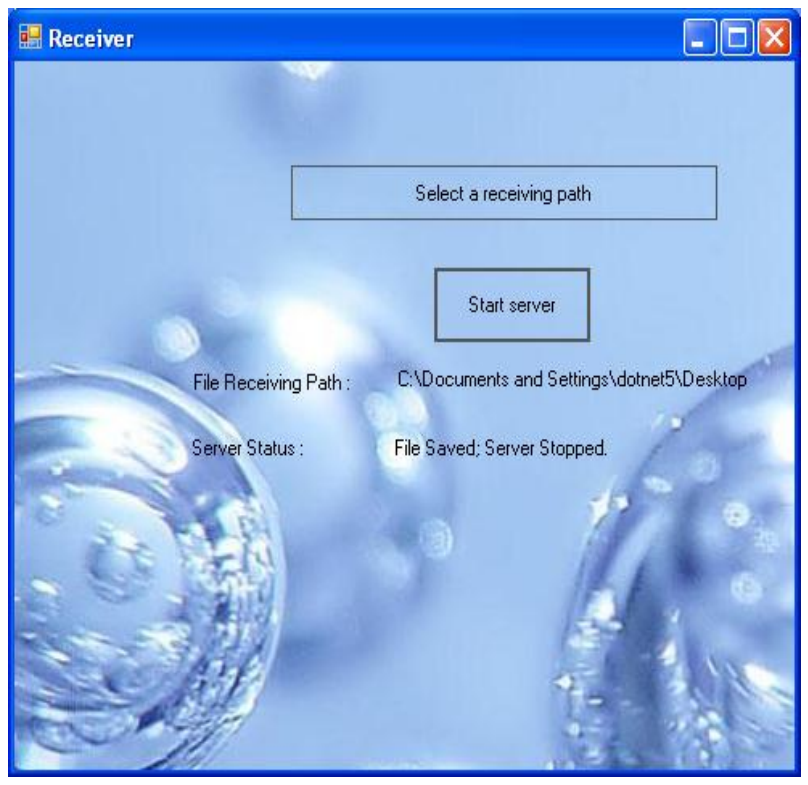

Fig 7.Select the Receiving path of sender 


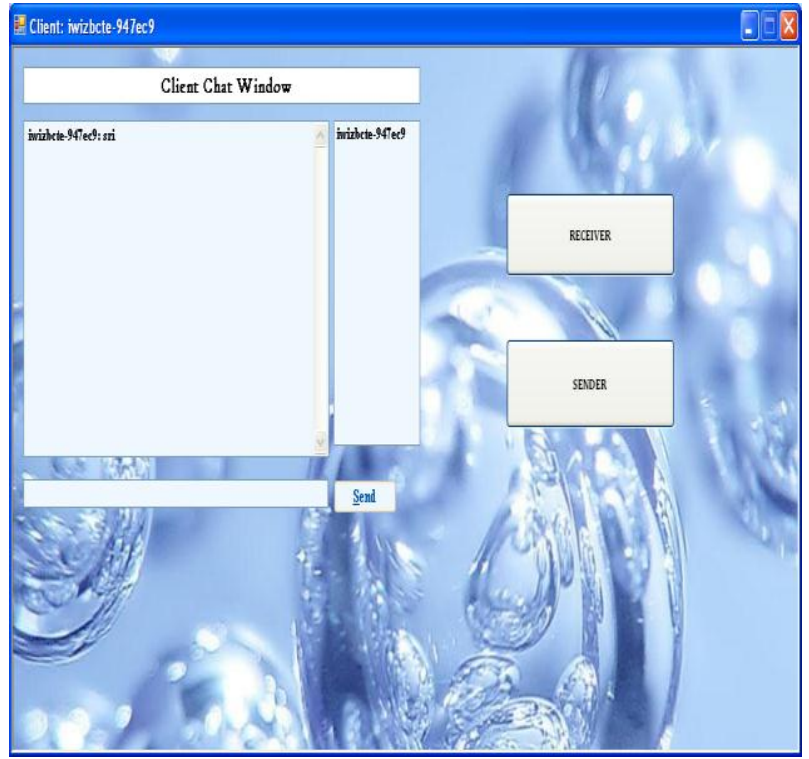

Fig 8.Chat window on receiver message

\section{CONCLUSION}

The Ballpark figure algorithms for broadcasting in multihop wireless networks. The algorithm for ONE-TO-ALL BROADCASTING gives a ballpark solution, and the algorithms for ALL-TO-ALL BROADCASTING give ballpark figure ratios of 20 and 34. Our model results show that in run through, these future schemes complete much better than the hypothetical bounce and accomplish up to 37 percent latency presentation development more than existing schemes.

\section{REFERENCES}

[1] I. Chlamtac and O. Weinstein, "The Wave Expansion Approach to Broadcasting in Multihop Radio Networks," IEEE Trans. Comm.,vol. 39, no. 3, pp. 426-433, Mar. 1991.

[2] S. Basagni, I. Chlamtac, and D. Bruschi, "A MobilityTransparent Deterministic Broadcast Mechanism for Ad Hoc Networks," IEEE/ACM Trans. Networking, vol. 7, no. 6, pp. 799-807, Dec. 1999.

[3]Z. Chen, C. Qiao, J. Xu, and T. Lee, "A Constant Approximation Algorithm for Interference Aware Broadcast in Wireless Networks,"Proc. IEEE INFOCOM, pp. 740-748, 2007.

[4] R. Tiwari, T.N. Dinh, and M.T. Thai, "On Approximation Algorithms for Interference-Aware Broadcast Scheduling in 2D and 3D Wireless Sensor Networks," Proc. Fourth Int'l Conf. Wireless Algorithms, Systems, and Applications (WASA '09), pp. 438-448, 2009.

[5] C. Ho, K. Obraczka, G. Tsudik, and K. Viswanath, "Flooding for Reliable Multicast in Multi-Hop Ad Hoc Networks," Proc. Third Int'l Workshop Discrete Algorithms and Methods for Mobile Computing and Comm., pp. 64-71, 1999.

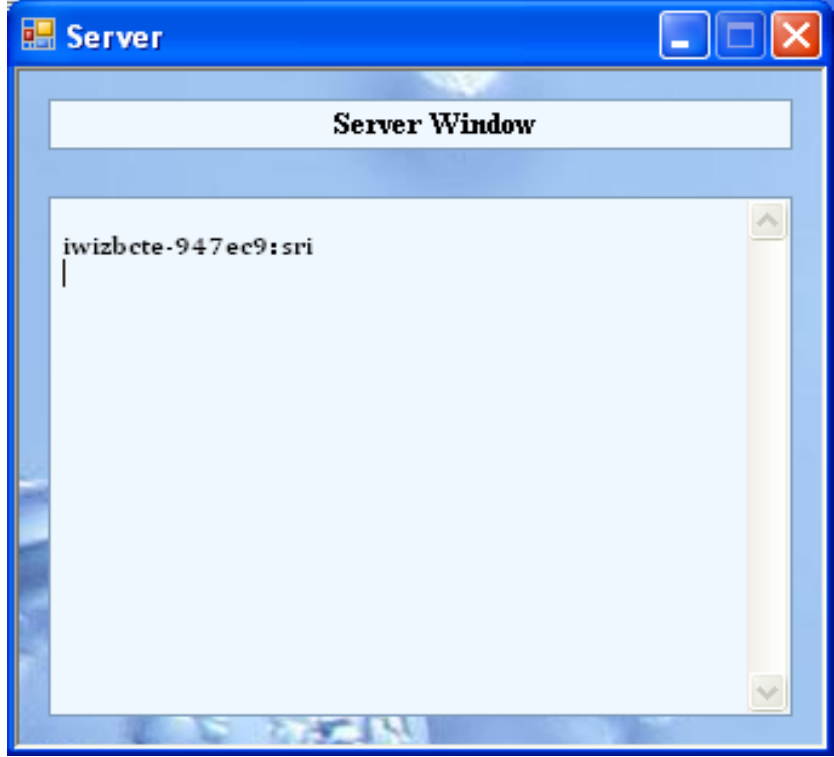

Fig 9.Receving the message on Server Window

[6] J. Jetcheva, Y. Hu, D. Maltz, and D. Johnson, “A Simple Protocol for Multicast and Broadcast in Mobile Ad Hoc Networks,” IETF Internet draft, July 2001.

[7] S.-Y. Ni, Y.-C. Tseng, Y.-S. Chen, and J.-P. Sheu, "The Broadcast Storm Problem in a Mobile Ad Hoc Network," Proc. ACM/IEEE MobiCom, pp. 151-162, 1999.

[8] Rajiv Gandhi, Yoo-Ah Kim, Seungjoon Lee, Jiho Ryu, Member, IEEE, and Peng-Jun Wan"Approximation Algorithms for Data Broadcast in Wireless Networks"IEEE Transactions on Mobilecomputing, vol. 11, no. 7, July 2012 